\title{
Arthroscopic Treatment for Osteoarthritic Knee
}

\author{
Chung Shik Shin, $\mathrm{MD}^{1}$ and Ju Hong Lee, $\mathrm{MD}, \mathrm{PhD}^{2}$ \\ ${ }^{1}$ Department of Orthopedic Surgery, Presbyterian Medical Center, Jeonju; ${ }^{2}$ Department of Orthopedic Surgery, Research Institute of Clinical Medicine of Chonbuk \\ National University Hospital, Chonbuk National University Medical School, Jeonju, Korea
}

\begin{abstract}
Arthroscopic treatment for osteoarthritis of the knee has been widely performed as one of the surgical options, in spite of persisting concerns regarding its efficacy. Arthroscopic debridement is a general term that is used to cover many procedures, including lavage, partial meniscectomy, removal of loose body, synovectomy, chondroplasty, removal of offending osteophytes, and/or microfracture. Recently, the role of arthroscopy in managing the osteoarthritic knee has been challenged by elusive consensus on its usefulness. Therefore, we review the available literatures for the arthroscopic intervention in knee osteoarthritis and summarized evidences for proper patient selection, which is a paramount factor to achieve the surgical goal of the arthroscopic treatment in osteoarthritic knee.
\end{abstract}

Key words: Knee, Osteoarthritis, Arthroscopic debridement, Patient selection.

\section{Introduction}

Osteoarthritis of the knee is characterized by wearing of the articular cartilage with subsequent damage to the bones, synovial membrane, and meniscus. The most common findings of osteoarthritis include pain, chronic effusion, osteophyte formation around the joint, subchondral sclerosis and cyst formation and degeneration or tear of the meniscus. Treatment options for osteoarthritis consist of conservative management, arthroscopic debridement, osteotomy and joint replacement. With the advancement in arthroscopic equipment and increased understanding on the pathophysiology of osteoarthritis in the 1970s, the advantages of minimally invasive arthroscopic techniques have become apparent. In 1990, Burks ${ }^{1)}$ described

Received July 16, 2012; Revised July 25, 2012; Accepted August 13, 2012. Correspondence to: Ju Hong Lee, MD.

Department of Orthopedic Surgery, Research Institute of Clinical Medicine of Chonbuk National University Hospital, Chonbuk National University Medical School, 20 Geonji-ro, Deokjin-gu, Jeonju 561-712, Korea.

Tel: +82-63-250-1760, Fax: +82-63-271-6538

Email: jhlee55@chonbuk.ac.kr

This is an Open Access article distributed under the terms of the Creative Commons Attribution Non-Commercial License (http://creativecommons.org/licenses/by-nc/3.0/) which permits unrestricted non-commercial use, distribution, and reproduction in any medium, provided the original work is properly cited. that the role of arthroscopy in the treatment of osteoarthritis of the knee is to allow identification and treatment of a local lesion while preserving the joint, which is accepted by most authors. Arthroscopic procedures for osteoarthritis of the knee include lavage, partial meniscectomy, chondroplasty, synovectomy, removal of loose body, removal of offending osteophytes, and adhesiolysis, which are performed in a proper combination according to the articular lesion type.

Arthroscopic treatment can be a good option for patients who want to delay arthroplasty to maintain some level of activities in that it has low morbidity and allows for future reconstruction. However, there is a controversy regarding the efficacy of the treatment, which we will discuss in this review article $e^{2-4)}$.

\section{Arthroscopic Debridement}

Arthroscopic debridement was first introduced for the treatment of osteoarthritis of the knee by Sprague ${ }^{5)}$ in 1981 and has been used for short-term symptom relief although its long-term efficacy ranges from $45 \%$ to $76 \%{ }^{6}$. Arthroscopic debridement consists of lavage, removal of loose body, partial meniscectomy, chondroplasty, synovectomy, removal of offending osteophytes, adhesiolysis, and joint insufflation. These procedures are helpful for short-term symptom relief in early arthritis, but ineffective for halting the progression of the disorder.

\section{Lavage}

In the past, lavage of the knee joint itself was considered helpful 
for improving symptoms by washing out proteolytic enzymes and loose tissues in the joint that cause pain. Currently, this procedure is considered to produce short-term relief or unsatisfactory results, and thus is viewed as a part of debridement ${ }^{7,8)}$.

\section{Meniscectomy}

Degenerative tears are commonly observed in osteoarthritic patients presenting with knee pain and loss of function. Considering that degenerative tears can occur secondary to arthritis, it is important to determine whether the symptoms are due to osteoarthritis or the tear of the meniscus.

Although meniscectomy is a common procedure, the load on the knee may increase by $45 \%$ even after a partial removal of the meniscus, which can accelerate the progression of arthritis and increase the necessity for arthroplasty. Pearse and Craig) found that patients with Outerbridge grade 4 cartilage degeneration and meniscus tear required additional surgery at a mean of 52 months after partial meniscectomy compared to the control group and concluded that the procedure does not stimulate the progression of arthritis. On the other hand, Rangger et al. ${ }^{10)}$ reported that osteoarthritis progressed in $38 \%$ of patients with medial meniscal tears and $24 \%$ of patients with lateral meniscal tears at a mean of 53.5 months after arthroscopic partial meniscectomy that was performed on a total of 284 patients.

Some studies have shown that partial mesniscectomy that has been generally considered more effective for early stage of osteoarthritis can be relatively helpful for advanced stage osteoarthritis. Lotke et al. ${ }^{11)}$ reviewed 101 patients (mean age, 55.6 years) who underwent medial meniscectomy for the mean of 10.8 years. The postoperative results were good in $90 \%$ of patients with normal preoperative radiographs, whereas only in $21 \%$ of the patients with more than moderate pre-existing degenerative changes. Therefore, they concluded that the preoperative level of degeneration is an important prognostic factor in meniscectomy, whereas age has no influence on the outcome. Further, they suggested that the major cause of symptoms should be identified prior to the procedure especially in elderly patients. McBride et al. ${ }^{12)}$ followed up 63 patients (mean age, 56.5 years) who underwent medial meniscectomy for the mean period of 35 months. In the study, satisfying results were obtained in $96 \%$ of the patients without arthroscopic evidence of degenerative changes, whereas in $65 \%$ of patients with degenerative changes. They concluded that the presence of preoperative degenerative changes affects postoperative results. Crevoisier et al. ${ }^{13)}$ reviewed 91 patients (mean age, 74 years) for the mean period of 4 years after arthroscopic meniscectomy. In the study, $81 \%$ of patients with Kellgren \& Lawrence (K-L) grade $0-2$ and $83 \%$ of the patients with Outerbridge grade 0-2 cartilage degeneration were satisfied with the postoperative results. Of the patients with K-L 3 ? 4 and those with Outerbridge 3-4, 55\% and 69\%, respectively, were satisfied and $45 \%$ and $37 \%$, respectively, underwent a further surgery 1-4 years afterwards. These results showed that preoperative cartilage degeneration had more influence on the treatment outcome than age. Although satisfactory results were obtained in $\geq 2 / 3$ of the patients with more than moderate osteoarthritis, it was not comparable to the results in young patients. Therefore, they suggested that partial meniscectomy should be performed with care in elderly patients regarding indications for surgery and postoperative outcomes.

On the other hand, Bonamo et al. ${ }^{14)}$ performed partial meniscectomy without any treatment for articular cartilage on 118 patients (mean age, 57 years) with Outerbridge grade 3-4 cartilage degeneration. For a mean of 3.3 years of follow-up, significant improvement was observed in $60 \%$ of the patients, but subjective satisfaction rate was $83 \%$. In 63 patients with Outerbridge grade 1-2 cartilage degeneration, significant improvement was found in $84 \%$. They reported that the poor prognostic factors were female gender, $\geq 60$ years of age, and pre-existing severe degenerative change and good results can also be expected in patients with Outerbridge grade 3-4 cartilage degeneration. Bin et al. ${ }^{15)}$ reviewed 68 patients (mean age, 63.3 years) for the mean period of 52 months after partial meniscectomy. The patients had Outerbridge grade 4 degenerative arthritis and medial meniscal tears preoperatively and were followed up for a mean period of 52 months. The postoperative Lysholm score and visual analogue scale score were improved in $82 \%$ and total knee arthroplasty (TKA) was required in $5.9 \%$ at a mean of 49.8 months postoperatively, indicating that partial meniscectomy could improve symptoms in Outerbridge grade 4 patients and delay TKA. This study shows that partial meniscectomy can also be helpful for advanced osteoarthritis.

\section{Osteophyte Excision}

Osteophytes are the most common radiographic findings in osteoarthritis. When articular cartilage is damaged, transforming growth factor- $\beta$ promotes cartilage repair resulting in endochondral ossification and osteophyte formation during which neurovascular invasion and innervation occur. Hyaline cartilage damage is the major pathology of arthritis. However, the local cause of pain in arthritis has not been clearly identified because cartilage does not contain nociceptive fibers. Cicuttini et al. ${ }^{16)}$ investigated the influence of joint space narrowing and 
osteophytes on knee pain in 250 middle-aged female patients. They reported that the presence of osteophytes was the more accurate predictor of knee pain, but did not clarify whether osteophyte itself was the cause of pain. Sengupta et al. ${ }^{17)}$ reported that the radiographic evidence of osteophytes was not related to the presence, location, and severity of pain contrary to their hypothesis. In contrast, Ozdemir et al. ${ }^{18)}$ reported that the size, location, and direction of osteophytes and the joint space narrowing were correlated with passive and active range of motion (ROM) in 84 osteoarthritic patients with chronic knee pain. Steadman et al. ${ }^{19)}$ and Fond et al. ${ }^{20)}$ reported satisfactory results of excision of osteophytes, which was the cause of limited knee extension and impingement. However, osteophyte excision was accompanied by other procedures for osteoarthritis in these studies making it difficult to determine the efficacy of the procedure.

\section{Chondroplasty}

The purpose of chondroplasty is not to promote regeneration but to prevent further mechanical damage to the remaining cartilage. Removal of the damaged area of the cartilage can be done precisely with the use of laser or high-frequency energy which is advantageous over shaver in terms of securing accessibility during surgery. Initially, laser was expected to be helpful for preventing damage to the intact cartilage and articular cartilage roughening, which can occur during mechanical shaving. However, severe abrasion on the intact cartilage, osteonecrosis, and high cost are increasingly recognized as problems of the use of laser. In particular, Holmium:Yag (Ho:YAG) laser that has been used in arthroscopic surgery because of its affinity for water theoretically appears to be useful for chondral lesion treatment. However, its efficacy has been in question because treatment success appears to be highly dependent on the kind and location of the lesion, technique, and experience of the surgeon.

Radiofrequency is an electrical current that produces heat when it passes through body tissues. It can be classified into monopolar (energy that is transmitted through tissues) and bipolar (energy that is transmitted through arthroscopic fluid). Recently, radiofrequency has been introduced in the treatment of articular cartilage lesions and considered effective for halting the progression of arthritis and functional impairment. It has been considered both economical and safe and used in various clinical settings. However, its efficacy for articular cartilage lesion treatment has remained controversial due to the risk of normal tissue damage and osteonecrosis. Kaplan and Uribe ${ }^{21)}$ reported that chondrocytes were viable without any alterations in collagen or microstructure after chondroplasty using bipolar radiofrequency. Turner et al. ${ }^{22}$ compared the results of bipolar radiofrequency ablation and mechanical shaving in an animal model three months postoperatively. There was less histological change and greater clinical improvement in the radiofrequency ablation group. Ko et al. ${ }^{23)}$ reported that bipolar radiofrequency can be useful for treating chondromalacic cartilage when used in a contact mode with a proper energy level and delivery time, although a long-term follow-up is necessary for accurate evaluation. Kaab et al. ${ }^{24)}$ recommended to use bipolar radiofrequency with caution because damage to normal cartilage was observed at 24 weeks after application in their animal model. Hogan and Diduch ${ }^{25}$ reported a case of progressive articular cartilage loss following bipolar radiofrequency treatment. Likewise, there is no consensus among authors on the efficacy of radiofrequency thermal ablation because it is dependent on the energy level, delivery time, characteristics of a lesion, and type of electrode.

\section{Bone Marrow Stimulation for Chondral Lesion}

Procedures for subchondral bone exposure caused by articular cartilage loss include arthroscopic drilling and abrasion arthroplasty, a 1-2 mm abrasion of the exposed subchondral bone in patients with a low level of activity and deformity. The procedures have been known to produce short-term symptomatic improvement. However, proper indications and contraindications, as well as evidence of efficacy have not been clearly established and durability of resulting fibrocartilage regeneration has been questionable.

In 1994, Rodrigo et al. ${ }^{26)}$ reported that microfracture that is based on the same principles as abrasion arthroplasty results in 1) less tissue damage by not causing heat necrosis of the subchondral bone and bone marrow and 2) decompression and pain relief if the depth and extent of fracture is adjusted to 3-4 $\mathrm{mm}$. Continuous passive motion following debridement and microfracture improved clinical outcomes and articular cartilage condition in $85 \%$ of the patients. However, the results have not been compared with those of debridement only. In addition, the procedure does not generally yield satisfying results in elderly patients with symptomatic osteoarthritis and extensive articular cartilage lesions.

\section{Discussion}

In 2002, Moseley et al. ${ }^{6}$ reported that arthroscopic debridement 
was no better than a placebo procedure in patients with $\geq \mathrm{K}-\mathrm{L}$ 3 ( $\geq$ moderate loss of joint space) in a placebo-controlled trial. Subsequently, the Arthroscopy Association of North America (AANA) officially announced that a proper arthroscopic procedure can be particularly beneficial to a specific group of osteoarthritic patients ${ }^{7}$. Afterwards, Jackson and Dieterichs ${ }^{2)}$ reported that arthroscopic debridement could be effective for early osteoarthritis based on the 4-6 years of follow-up results. Aaron et al. ${ }^{27)}$ observed improvements in $90 \%$ of patients with early osteoarthritis, whereas in $25 \%$ of the patients with advanced osteoarthritis during the 34 months of follow-up period after arthroscopic debridement. Steadman et al. ${ }^{19)}$ reviewed the results of joint insufflation and conventional arthroscopic debridement procedures in patients with $\geq \mathrm{K}$-L 3 osteoarthritis for a minimum 2 years of follow-up. Satisfying results were obtained in $71 \%$ of patients, indicating that debridement would improve joint function and level of activity. On the other hand, Siparsky et al. ${ }^{28}$ reviewed the current literature and concluded that arthroscopic debridement may have some level of utility in knees with low-grade osteoarthritis, but it cannot be a routine treatment for all osteoarthritic patients. Kirkley et al. ${ }^{3)}$ assessed the efficacy of arthroscopic debridement using a study design that compensates for some shortcomings of a Moseley's study and found that arthroscopic debridement was not advantageous over optimized physical and medical therapy in patients with $\geq \mathrm{K}-\mathrm{L} 2$ osteoarthritis. Litchfield et al. ${ }^{4)}$ performed arthroscopic treatment on the knees with moderate osteoarthritis in a similar method as Steadman and reported that the procedure had no additional benefits over conservative treatment. These studies indicate that arthroscopic debridement is a procedure still surrounded by controversy.

The prevalence of a meniscal tear is high in middle-aged and elderly patients with $\geq \mathrm{K}-\mathrm{L} 3$ osteoarthritis and $61 \%$ of those with MRI evidence of a meniscal tear do not present with pain $^{29)}$. Bhattacharyya et al. ${ }^{30)}$ reported that MRI showed meniscal tears in $91 \%$ of the osteoarthritic patients with $\geq 45$ years of age and $76 \%$ of them had no symptoms. Therefore, they concluded that meniscal tears are common in osteoarthritic knees regardless of the presence of symptoms. In addition, the presence of a tear was neither associated with the level of pain nor had a significant influence on the knee function. Therefore, a meniscal tear may not be the cause of knee pain and it is difficult to determine the cause of pain in knees with both osteoarthritis and meniscal tears. In particular, a meniscal tear is not the major cause of pain in most of the cases with K-L 3-4 osteoarthritis. In general, the need for orthopedic surgery is determined based
Table 1. Favorable Prognostic Factors

1. Localized symptoms of less than 6 months

2. Mechanical symptoms (catching or locking)

3. Clinical sign

- Small or no effusion, localized tenderness, and positive meniscal stress test

4. ROM: $<10^{\circ}$ of flexion contracture $\&>120^{\circ}$ flexion

5. Weight bearing view

$<3 \mathrm{~mm}$ joint space narrowing, within physiological valgus of $4^{\circ}$ to $9^{\circ}$ in mechanical alignment

6. Age: $<70 \mathrm{yr}$

7. BMI: $<30$

ROM: range of motion; BMI: body mass index.

on the symptoms, quality of life assessment, and disabilities, albeit difficult to quantify. However, anatomical and MRI abnormalities are not related to the symptoms of joint disorders and correction of abnormalities does not necessarily result in functional improvement. Therefore, Marx ${ }^{31)}$ emphasized the need to individualize decision making on surgery for osteoarthritis, although arthroscopic surgery remains appropriate when osteoarthritis is not believed to be the primary cause of knee pain. The prognostic factors include clinical symptoms in the affected joint, mechanical symptoms, duration of morbidity, presence of a meniscal tear, ROM, lower limb alignment, joint space narrowing, age, weight, and smoking. Favorable prognostic factors are listed in Table 1.

The National Institute for Clinical Excellence (NICE) provided updated guidelines for arthroscopic treatment of osteoarthritis in 2007 where the importance of proper patient selection was emphasized because its efficacy is uncertain and the procedure was recommended for the knees with locking associated with intraarticular loose bodies or meniscus tears. According to the 2008 osteoarthritis treatment guidelines set by the American Association of Orthopedic Surgery (AAOS), arthroscopic debridement or lavage is not recommended for patients with primary diagnosis of symptomatic osteoarthritis of the knee (Recommendation 18), whereas the partial meniscectomy or loose body removal can be an option in the knees with mechanical symptoms of a torn meniscus or loose body, such as catching or locking (Recommendation 19) ${ }^{32)}$.

The indications for arthroscopic debridement based on the review of clinical studies on patient selection ${ }^{27,33-39)}$ can be summarized as follows: joint effusion, localized joint line tenderness, acute or aggravating mechanical symptoms, such as catching or locking, development or aggravation of symptoms 
related to a specific injury mechanism, intraarticular loose body confirmed with imaging, and early stage degenerative arthritis without appearance of malalignment, severe joint space narrowing ( $\leq 5 \mathrm{~mm}$ ), and multiple or large-sized osteophytes. Weight-bearing radiographs, medical records, and physical examination results should be thoroughly evaluated. In addition, care should be taken to the fact that MRI has low specificity and high sensitivity for osteoarthritis of the knee. The patient should be fully aware that the goal of arthroscopic treatment is not to cure the disorder but to relieve pain. Arthroscopic treatment can contribute to symptomatic improvement in patients with advanced osteoarthritis if severe acute pain related to catching or locking occurs in the affected compartment, symptoms related to meniscal tear, loose body, or an articular cartilage flap exist in an intact compartment, or patellofemoral impingement, loss of extension, bursitis, synovitis, intraarticular ligament damage, which is caused by a large-sized osteophyte is present.

\section{Conclusions}

The efficacy of arthroscopic treatment for osteoarthritis of the knee is limited by poor natural history of osteoarthritis. For successful treatment results, care should be taken to detailed medial history including related symptoms and signs, thorough physical examination, and clinical findings on weight-bearing radiographs for proper patient selection. Furthermore, the surgeon should provide sufficient information on the benefits and risks of the treatment prior to surgery in order to help the patient better understand the implications of the surgery.

\section{References}

1. Burks RT. Arthroscopy and degenerative arthritis of the knee: a review of the literature. Arthroscopy. 1990;6:43-7.

2. Jackson RW, Dieterichs C. The results of arthroscopic lavage and debridement of osteoarthritic knees based on the severity of degeneration: a 4- to 6-year symptomatic followup. Arthroscopy. 2003;19:13-20.

3. Kirkley A, Birmingham TB, Litchfield RB, Giffin JR, Willits KR, Wong CJ, Feagan BG, Donner A, Griffin SH, D’Ascanio LM, Pope JE, Fowler PJ. A randomized trial of arthroscopic surgery for osteoarthritis of the knee. N Engl J Med. 2008;359:1097-107.

4. Litchfield RB, Kirkley A, Brimingham T, Giffin R, Willits K, Feagan B, Donner A, Griffin SH, D’Ascanio L, Fowler PJ. A randomized trial comparing arthroscopic surgery to non- surgical care for knee osteoarthritis (oa (ss-55). Arthroscopy 2009;25:e30.

5. Sprague NF 3rd. Arthroscopic debridement for degenerative knee joint disease. Clin Orthop Relat Res. 1981;(160):11823.

6. Moseley JB, O'Malley K, Petersen NJ, Menke TJ, Brody BA, Kuykendall DH, Hollingsworth JC, Ashton CM, Wray NP. A controlled trial of arthroscopic surgery for osteoarthritis of the knee. N Engl J Med. 2002;347:81-8.

7. Livesley PJ, Doherty M, Needoff M, Moulton A. Arthroscopic lavage of osteoarthritic knees. J Bone Joint Surg Br. 1991;73:922-6.

8. Edelson R, Burks RT, Bloebaum RD. Short-term effects of knee washout for osteoarthritis. Am J Sports Med. 1995;23:345-9.

9. Pearse EO, Craig DM. Partial meniscectomy in the presence of severe osteoarthritis does not hasten the symptomatic progression of osteoarthritis. Arthroscopy. 2003;19:963-8.

10. Rangger C, Klestil T, Gloetzer W, Kemmler G, Benedetto KP. Osteoarthritis after arthroscopic partial meniscectomy. Am J Sports Med. 1995;23:240-4.

11. Lotke PA, Lefkoe RT, Ecker ML. Late results following medial meniscectomy in an older population. J Bone Joint Surg Am. 1981;63:115-9.

12. McBride GG, Constine RM, Hofmann AA, Carson RW. Arthroscopic partial medial meniscectomy in the older patient. J Bone Joint Surg Am. 1984;66:547-51.

13. Crevoisier X, Munzinger U, Drobny T. Arthroscopic partial meniscectomy in patients over 70 years of age. Arthroscopy. 2001;17:732-6.

14. Bonamo JJ, Kessler KJ, Noah J. Arthroscopic meniscectomy in patients over the age of 40. Am J Sports Med. 1992;20:4228.

15. Bin SI, Lee SH, Kim CW, Kim TH, Lee DH. Results of arthroscopic medial meniscectomy in patients with grade IV osteoarthritis of the medial compartment. Arthroscopy. 2008;24:264-8.

16. Cicuttini FM, Baker J, Hart DJ, Spector TD. Association of pain with radiological changes in different compartments and views of the knee joint. Osteoarthritis Cartilage. 1996;4:143-7.

17. Sengupta M, Zhang YQ, Niu JB, Guermazi A, Grigorian M, Gale D, Felson DT, Hunter DJ. High signal in knee osteophytes is not associated with knee pain. Osteoarthritis Cartilage. 2006;14:413-7.

18. Ozdemir F, Tukenmez O, Kokino S, Turan FN. How do 
marginal osteophytes, joint space narrowing and range of motion affect each other in patients with knee osteoarthritis. Rheumatol Int. 2006;26:516-22.

19. Steadman JR, Ramappa AJ, Maxwell RB, Briggs KK. An arthroscopic treatment regimen for osteoarthritis of the knee. Arthroscopy. 2007;23:948-55.

20. Fond J, Rodin D, Ahmad S, Nirschl RP. Arthroscopic debridement for the treatment of osteoarthritis of the knee: 2- and 5-year results. Arthroscopy. 2002;18:829-34.

21. Kaplan L, Uribe JW. The acute effects of radiofrequency energy in articular cartilage: an in vitro study. Arthroscopy. 2000;16:2-5.

22. Turner AS, Tippett JW, Powers BE, Dewell RD, Mallinckrodt $\mathrm{CH}$. Radiofrequency (electrosurgical) ablation of articular cartilage: a study in sheep. Arthroscopy. 1998;14:585-91.

23. Ko SH, Kim KS, Choi YS, Chung YY, Song JY, Park BH, Choi JB, Kim DJ, Kim YS, Lee JH. The effects of radiofrequency energy on human articular cartilage: the effect of power setting and mode of treatment on chondrocyte viability. J Korean Orthop Assoc. 2003;38:757-62.

24. Kaab MJ, Bail HJ, Rotter A, Mainil-Varlet P, apGwynn I, Weiler A. Monopolar radiofrequency treatment of partialthickness cartilage defects in the sheep knee joint leads to extended cartilage injury. Am J Sports Med. 2005;33:1472-8.

25. Hogan CJ, Diduch DR. Progressive articular cartilage loss following radiofrequency treatment of a partial-thickness lesion. Arthroscopy. 2001;17:E24.

26. Rodrigo JJ, Steadman JR, Silliman JF, Fulstone HA. Improvement of full-thickness chondral defect healing in the human knee after debridement and microfracture using continuous passive motion. Am J Knee Surg. 1994;7:109-16.

27. Aaron RK, Skolnick AH, Reinert SE, Ciombor DM. Arthroscopic debridement for osteoarthritis of the knee. J Bone Joint Surg Am. 2006;88:936-43.

28. Siparsky P, Ryzewicz M, Peterson B, Bartz R. Arthroscopic treatment of osteoarthritis of the knee: are there any evidence-based indications? Clin Orthop Relat Res.
2007;455:107-12.

29. Englund M, Guermazi A, Gale D, Hunter DJ, Aliabadi P, Clancy M, Felson DT. Incidental meniscal findings on knee MRI in middle-aged and elderly persons. N Engl J Med. 2008;359:1108-15.

30. Bhattacharyya T, Gale D, Dewire P, Totterman S, Gale ME, McLaughlin S, Einhorn TA, Felson DT. The clinical importance of meniscal tears demonstrated by magnetic resonance imaging in osteoarthritis of the knee. J Bone Joint Surg Am. 2003;85:4-9.

31. Marx RG. Arthroscopic surgery for osteoarthritis of the knee? N Engl J Med. 2008;359:1169-70.

32. American Academy of Orthopaedic Surgeons (AAOS). Treatment of osteoarthritis of the knee (non-arthroplasty) [Internet]. Rosemont (IL): AAOS; c2012 [cited 2012 Jul 13]. Available from: http:// http://www.aaos.org/research/ guidelines/oakguideline.pdf.

33. Hunt SA, Jazrawi LM, Sherman OH. Arthroscopic management of osteoarthritis of the knee. J Am Acad Orthop Surg. 2002;10:356-63.

34. Felson DT. Arthroscopy as a treatment for knee osteoarthritis. Best Pract Res Clin Rheumatol. 2010;24:47-50.

35. Dearing J, Nutton RW. Evidence based factors influencing outcome of arthroscopy in osteoarthritis of the knee. Knee. 2008;15:159-63.

36. Stuart MJ, Lubowitz JH. What, if any, are the indications for arthroscopic debridement of the osteoarthritic knee? Arthroscopy. 2006;22:238-9.

37. Spahn G, Muckley T, Kahl E, Hofmann GO. Factors affecting the outcome of arthroscopy in medial-compartment osteoarthritis of the knee. Arthroscopy. 2006;22:1233-40.

38. Howell SM. The role of arthroscopy in treating osteoarthritis of the knee in the older patient. Orthopedics. 2010;33:652.

39. Sgaglione NA, Chen E, Bert JM, Amendola A, Bugbee WD. Current strategies for nonsurgical, arthroscopic, and minimally invasive surgical treatment of knee cartilage pathology. Instr Course Lect. 2010;59:157-80. 